\title{
La utilización de las tecnologías de la información y la comunicación (TIC) por docentes de Optometría: propuesta de software educativo
}

\author{
Use of Information and Communication Technologies \\ (ICT) by Optometry teachers: Proposal of Educational \\ Software
}

\author{
Fabrina Anyelit Aburto Garcés \\ Universidad Nacional Autónoma de Nicaragua \\ Managua, Nicaragua \\ dra.faburto@yahoo.com
}

Recibido: 14/08/2019 Aceptado: 18/11/2019

\begin{abstract}
Resumen. El objetivo de este estudio es analizar el uso que les dan los docentes universitarios de la carrera de Optometría Médica a las tecnologías de la información y la comunicación (TIC). El enfoque metodológico fue mixto, para lo cual se diseñaron instrumentos cuantitativos y cualitativos como encuestas, entrevistas a profundidad y observación del aula de clase. Se identificaron las siguientes categorías analíticas: tipo y usos que les dan los docentes a las TIC; beneficios que promueven en el aprendizaje y condiciones que favorecen su uso. Los principales recursos tecnológicos que utilizan los docentes son computadora, Power Point, videos y redes sociales. Las TIC en el aprendizaje mejoran la comprensión del tema y la asimilación de contenidos. Los docentes de la carrera de Optometría Médica no han recibido capacitación en el uso de las TIC. La infraestructura tecnológica con que cuenta la carrera resulta insuficiente para poder aplicar las tecnologías en el aula de clase. De manera que se propone la creación de un software educativo para la carrera de Optometría Médica.
\end{abstract}

Palabras clave: usos de las TIC, docentes universitarios, proceso enseñanza-aprendizaje, software educativo. 


\begin{abstract}
This study aims to analyze how university professors use information and communication technologies (TIC) in the career of Medical Optometry. The methodological approach implemented quantitative and qualitative instruments, such as surveys, in-depth interviews, and observation in the classroom. The following analytical categories were identified: types of TICs and how teachers use them, and benefits that promote learning and conditions that favor their use. The main technological resources that teachers use are PowerPoint, computers, videos, and social networks. TICs used in learning improve understanding of topics and assimilation of content. The teachers of the career of Medical Optometry have not received training in using TICs. The technological infrastructure of the career is insufficient to apply the technologies in the classroom. So it is proposed to create educational software for the career of Medical Optometry.
\end{abstract}

Keywords: uses of TIC, university teachers, teaching-learning process, educational software.

\title{
Introducción
}

Actualmente, las tecnologías de la información y la comunicación (TIC) han alcanzado un notable desarrollo en todos los ámbitos. A nivel educativo vienen a contribuir y mejorar los procesos de enseñanza-aprendizaje. Como lo expresa la Unesco: "Las TIC constituyen una herramienta útil que permite diversificar la enseñanza y el aprendizaje, favorecen el desarrollo de nuevas prácticas educativas pertinentes y eficaces, lo que incluye fortalecer el protagonismo que tienen los docentes en los cambios educativos" (2013, p. 19).

Conceptualizando el término TIC, Cabero (1998) expone que las nuevas tecnologías de la información y la comunicación giran en torno a tres medios básicos: la informática, la microelectrónica y las telecomunicaciones. De manera que cuando hablamos de nuevas tecnologías nos referimos no solo a los equipos (hardware) que hacen posible la comunicación, sino también al desarrollo de aplicaciones (software).

Marqués Graell (2008) clasifica las TIC en medios hipertextos, medios hipermedia, medios multimedia, medios audiovisuales, medios comunicativos, programas informáticos y hardware informático.

Los jóvenes de hoy son nativos digitales, influenciados cada vez más por las TIC. La mayoría de ellos quieren aprender utilizando estas tecnologías con las cuales ya están familiarizados, por lo que la inclusión en el proceso de aprendizaje genera grandes beneficios para los estudiantes, entre los que se destacan: el desarrollo de habilidades de pensamiento crítico y la construcción 
URL: http://www.revistas.una.ac.cr/index.php/dialogo/index

CORREO ELECTRÓNICO: universidadendialogo@una.cr

DOI: https://doi.org/10.15359/udre.10-1.7

de conocimientos de manera creativa y dinámica (Ferreiro, 2010). De igual manera, la incorporación de las TIC en el aula de clase permite que los docentes impulsen nuevas formas de enseñar mediante la creación de escenarios de aprendizaje innovadores.

Dentro de los múltiples beneficios que ofrecen las TIC en el ámbito educativo están los siguientes: para el estudiante sirven de elemento motivador, interdisciplinario y de fácil acceso, propician la autonomía, el liderazgo y el trabajo colaborativo; para el docente implican múltiples recursos educativos para usarse en el aula, favorecen la comunicación con los estudiantes (Fernández, 2010). Sin embargo, las TIC no solo propician la actividad independiente, sino también la actividad con otros en tiempo real (sincrónica) y en tiempo diferido (asincrónica), lo que facilita el trabajo en equipo cooperativo y las relaciones sociales a distancia.

Lo anterior está apoyado en diversos estudios que evalúan la incorporación de las TIC en el sistema educativo, donde "los resultados más relevantes reportados en distintas latitudes coinciden en que los alumnos experimentan un aprendizaje significativo a través del uso apropiado de las TIC" (Pulido y Zambrano, 2010, p. 167).

Alrededor de esta temática existen diversas investigaciones, Vélez Figueroa (2012) plantea en su estudio sobre estrategias de enseñanza implementadas por los docentes al utilizar las TIC en su práctica pedagógica que los docentes utilizan diversos recursos tecnológicos como videos, computador para hacer presentaciones de diapositivas, programas interactivos o software de aplicación, simuladores, fotografías, podcast; sin embargo, hay resistencia en incorporar otros recursos tecnológicos.

Todos estos recursos son utilizados principalmente para transmitir información, apoyar las explicaciones del profesor y motivar a estudiantes, no se aplican para promover la participación activa del estudiante ni para la creación de conocimiento, aspectos que deben ser fortalecidos.

El estudio de maestría realizado por Esteban Alberto Ruiz Acevedo, Análisis de la incidencia de las tecnologías informáticas de la computación, en el proceso de enseñanza-aprendizaje que realiza el docente de Ciencias Sociales, con los alumnos de segundo año de magisterio de la Escuela Normal "Mirna Mairena Guadamuz" del municipio de Estelí, durante el I semestre del año 2012, refleja cómo las tecnologías informáticas de la computación tienen incidencia directa en el proceso de enseñanza-aprendizaje de las Ciencias Sociales. Se encontraron 
Revista Universidad en Diálogo • Vol. 10, N. ${ }^{\circ}$ 1, Enero-Junio, 2020 • 109-126

ISSN 2215-2849 • EISSN: 2215-4752

URL: http://www.revistas.una.ac.cr/index.php/dialogo/index Correo eleCtrónico: universidadendialogo@una.cr

DOI: https://doi.org/10.15359/udre.10-1.7

algunos obstáculos que impiden su uso para ser aprovechadas satisfactoriamente en el desarrollo de la clase, como la falta de capacitaciones que garanticen el aprovechamiento adecuado de estas herramientas. Las tecnologías informáticas de la computación existen, pero no se hace un uso adecuado de ellas en la tarea pedagógica, los fines para los que se utilizan son meramente administrativos, así se pierde la oportunidad de ser aprovechadas adecuadamente para lograr aprendizajes significativos y pertinentes (Ruiz, 2012).

Otro estudio, realizado por Karen García Loáisiga, Rol del docente y del estudiante universitario en el uso de las tecnologías de información como recursos didácticos en el proceso enseñanza-aprendizaje del tercer año de la carrera de Pedagogía con Mención en Educación Primaria de la Facultad de Educación e Idiomas de la UNAN-Managua, II semestre 2012, analiza el rol del docente y del estudiante universitarios en el uso de las TIC como recurso didáctico en el proceso de enseñanza-aprendizaje del grupo seleccionado. La investigación muestra que una minoría de los docentes y estudiantes informantes utiliza recursos TIC en el proceso de enseñanza-aprendizaje; la mayoría hace uso de recursos tradicionales, es decir, hacen uso escaso de los recursos TIC, debido al poco conocimiento y al poco acceso a estos recursos. Aun así, valoraron su importancia como herramienta para uso propio y como recurso didáctico en el proceso de enseñanza-aprendizaje (García, 2012).

Uno de los propósitos de la incorporación de las TIC a la educación escolar es aprovechar la potencialidad de estas tecnologías para impulsar nuevas formas de aprender y enseñar. No se trata ya de utilizar las TIC para hacer lo mismo pero mejor, con mayor rapidez y comodidad o incluso con mayor eficacia, sino para hacer cosas diferentes, para poner en marcha procesos de aprendizaje y de enseñanza que no serían posibles en ausencia de las TIC (Coll, 2011).

En este sentido, los docentes se ven en la necesidad de implementar la tecnología en su práctica pedagógica; sin embargo, no todos están haciendo uso adecuada de la misma. El problema radica en que muchos profesores utilizan las tecnologías como sustituto de los recursos tradicionales de enseñanza sin renovar sus estrategias metodológicas, con lo cual se pierden los innumerables beneficios que brindan las tecnologías. Es insuficiente que los docentes y estudiantes tengan acceso a las TIC, lo importante radica en el uso efectivo que hagan de ellas y que las incorporen de manera natural en las prácticas académicas (Hernández et al., 2014).

La UNAN Managua en su modelo educativo también contempla el uso de la tecnología en todo el quehacer docente, aplicándola como un recurso para favorecer el aprendizaje de los estudiantes (Modelo Educativo Normativa y 
URL: http://www.revistas.una.ac.cr/index.php/dialogo/index

CORREO ELECTRÓNICO: universidadendialogo@una.cr

DOI: https://doi.org/10.15359/udre.10-1.7

Metodología para la Planificación Curricular Unan-Managua, 2011). En este sentido, la carrera de Optometría Médica contempla en sus planes de clase la incorporación de la tecnología, adquiriendo gran importancia, puesto que el estudiante debe adquirir habilidades prácticas que muchas veces no es posible explicar a través de medios tradicionales de enseñanza. A través de la utilización de diversos recursos tecnológicos como videos, simuladores, Interne, entre otros, los estudiantes pueden comprender mejor los contenidos teóricos y vincular la teoría con la práctica para lograr aprendizajes significativos.

El propósito de esta investigación es analizar el uso que les dan los docentes de la carrera de Optometría Médica a las tecnologías de la información y la comunicación para favorecer el aprendizaje de los estudiantes de primero a quinto año en la Facultad de Ciencias Médicas, Unan-Managua, durante el I semestre 2017. En este estudio se describen los tipos de recursos tecnológicos que utilizan los docentes en el aula de clase, los beneficios que brindan al proceso de enseñanza-aprendizaje, así como las condiciones que favorecen la implementación de las tecnologías por los profesores.

El presente estudio es realizado siguiendo un enfoque mixto, donde se hace uso de la metodología cualitativa con el fin de lograr una perspectiva holística y profunda de la investigación.

Dentro de los resultados se encontró que los recursos tecnológicos más utilizados por los docentes son: computadora, Power Point, data show, memoria USB, videos de YouTube y redes sociales; recursos de más fácil manejo y a los cuales tienen mayor acceso. La infraestructura tecnológica con que cuenta la carrera está constituida por equipos básicos como data show, parlantes, extensiones, computadoras e Internet para los docentes. Sin embargo, estos recursos resultan insuficientes para poder aplicar las TIC en el aula de clase. Los estudiantes manifiestan que el uso de las TIC favorece el aprendizaje en tanto mejora la comprensión del tema, facilita la asimilación de contenidos de aprendizaje, favorece la retroalimentación y la comunicación entre profesores y estudiantes. Los docentes de la carrera de Optometría Médica no han recibido capacitación en el uso de las TIC como herramienta para favorecer el aprendizaje de los estudiantes. La mayoría tiene conocimientos básicos que han aprendido empíricamente.

\section{Material y método}

El estudio se basó en un enfoque mixto de tipo descriptivo y corte transversal. Se utilizó la triangulación metodológica y de fuentes, lo que permite minimizar la varianza de error, ayuda a superar las limitaciones de las propuestas de un 
método único y facilita el abordaje de la realidad desde distintos enfoques, eliminando sesgos (Hernández et al., 2006).

El escenario de la investigación fue la Universidad Nacional Autónoma de Nicaragua, Recinto Universitario Rubén Darío, dentro de este se encuentra la Facultad de Ciencias Médicas a la cual pertenece la carrera de Optometría Médica ubicada en el pabellón 64, que es donde se realizó el estudio.

La población la conformaron diez profesores que integran la planta docente de la carrera de Optometría Médica, 161 estudiantes de primero a quinto año, un ingeniero responsable del laboratorio de informática de la Facultad de Ciencias Médicas y el coordinador de la carrera.

Para el estudio se seleccionó una muestra de docentes y estudiantes por conveniencia a criterio del investigador, quedando una muestra de siete docentes.

\section{Criterios de inclusión de los docentes}

- Docentes que imparten las asignaturas de Lentes de Contacto, Optometría I, Optometría II, Fisiología General, Óptica Geométrica, Bioquímica, Psicología de la Salud Visual.

- Docentes que tienen más de dos años de experiencia docente.

\section{Criterios de exclusión de los docentes}

- Docentes que participan en las prácticas de profesionalización que se llevan a cabo en las clínicas que están fuera de la universidad.

La muestra de estudiantes fue de 114; para calcular el tamaño de la muestra de los estudiantes se utilizó el muestreo en dos etapas, el primero comprende el cálculo de la muestra total de la población y en un segundo momento el cálculo por estrato, para ello se utilizó el modelo matemático $n=\frac{N Z_{a}^{2} p q}{d^{2}(N-1)+Z_{a}^{2} p q}$ ecuación $\mathrm{N}^{\circ} 1$, que es el modelo que se aplica conociendo la población; para este caso se consideró la estimación del error del $5 \%$ y un $95 \%$ en el nivel de confianza. 
URL: http://www.revistas.una.ac.cr/index.php/dialogo/index

CORREO ELECTRÓNICO: universidadendialogo@una.cr

DOI: https://doi.org/10.15359/udre.10-1.7

Sustituyendo los valores en la ecuación $\mathrm{N}^{\circ} 1$ se encontraron los siguientes resultados $n=\frac{161(1.96)^{2}(0.5)(0.5)}{(0.05)^{2}(161)+(1.96)^{2}(0,5)(0.5)}$

Donde n es 113.7 aproximadamente 114 estudiantes.

Para encontrar la muestra de cada estrato se aplicó la ecuación $n_{i}=\frac{N_{i}}{N} n_{I}$ ecuación $\mathrm{N}^{\circ} 2$, sustituyendo los valores en la ecuación $\mathrm{N}^{\circ} 2$ se obtuvieron los siguientes datos:

Tabla 1

Estudiantes seleccionados por año cursado

\begin{tabular}{|l|c|c|c|}
\hline Estudiantes & Población & Muestra & Porcentaje (\%) \\
\hline Primer año & 50 & 36 & 70 \\
\hline Segundo año & 29 & 20 & 69 \\
\hline Tercero año & 23 & 16 & 69 \\
\hline Cuarto año & 23 & 16 & 69 \\
\hline Quinto año & 36 & 26 & 64 \\
\hline Total & 161 & 114 & 71 \\
\hline
\end{tabular}

Fuente: elaboración propia

La escogencia de los estudiantes de cada año que iban a participar en el estudio se realizó de manera aleatoria, mediante el procedimiento de tómbola.

Debido a la naturaleza mixta del estudio, se diseñaron instrumentos cuantitativos y cualitativos de recopilación de datos; se elaboraron entrevistas y encuestas dirigidas a docentes y estudiantes de primero a quinto año de la carrera de Optometría Médica, entrevistas semiestructuradas al ingeniero encargado del laboratorio de informática y al coordinador de carrera, así mismo se realizó una discusión de grupo focal con ocho estudiantes de cuarto y quinto año de la carrera de Optometría Médica seleccionados por conveniencia, porque eran los que tenían más tiempo de estar en la carrera y por lo tanto conocían a todos los docentes que impartían clases. Finalmente, se realizó una observación directa del aula de clase de cada uno de los docentes que formaron parte de la muestra. 
Revista Universidad en Diálogo • Vol. 10, N. ${ }^{\circ}$ 1, Enero-Junio, 2020 • 109-126

ISSN 2215-2849 • EISSN: 2215-4752

URL: http://www.revistas.una.ac.cr/index.php/dialogo/index CoRreo ElECTRÓNICO: universidadendialogo@una.cr

DOI: https://doi.org/10.15359/udre.10-1.7

Para verificar la validez de los instrumentos se realizó primeramente la evaluación de expertos, dos profesores de Pedagogía de la UNAN-Managua con alta trayectoria en su campo y un especialista en investigación científica. Una vez validados los instrumentos por los expertos, se realizó un pilotaje con estudiantes y docentes voluntarios que no forman parte de la muestra, aplicándose las encuestas a cinco estudiantes y tres docentes.

Para el análisis de los datos obtenidos se procesó de manera independiente cada instrumento. Los resultados de las encuestas a docentes y estudiantes fueron procesados en el programa estadístico SSPF para su posterior análisis, utilizando la estadística descriptiva para obtener datos de frecuencias y porcentajes. Las entrevistas a docentes, al grupo focal, al coordinador de carrera y al responsable del laboratorio se procesaron mediante una matriz de doble entrada, con el propósito de identificar semejanzas y diferencias de la información suministrada, lo que permitió cruzar la información emitida por los informantes a través de la triangulación de datos.

\section{Análisis y discusión de resultados}

\section{Tipos de recursos tecnológicos que usan los docentes}

Los recursos tecnológicos más utilizados por los docentes en el aula de clase son las computadoras, seguidas del Power Point y la memoria USB; además, se apoyan en otros recursos como Internet, videos, correo electrónico y teléfono celular. Por otro lado, los recursos que tienen menor frecuencia de uso: pizarra electrónica, plataformas virtuales, videoconferencia y simuladores, posiblemente por la complejidad en su funcionamiento, ya que la utilización de estos recursos requiere mayor conocimiento de parte de los docentes (Ver figura 1).

Opinión del estudiante sobre los tipos de recursos tecnológicos que usan los docentes

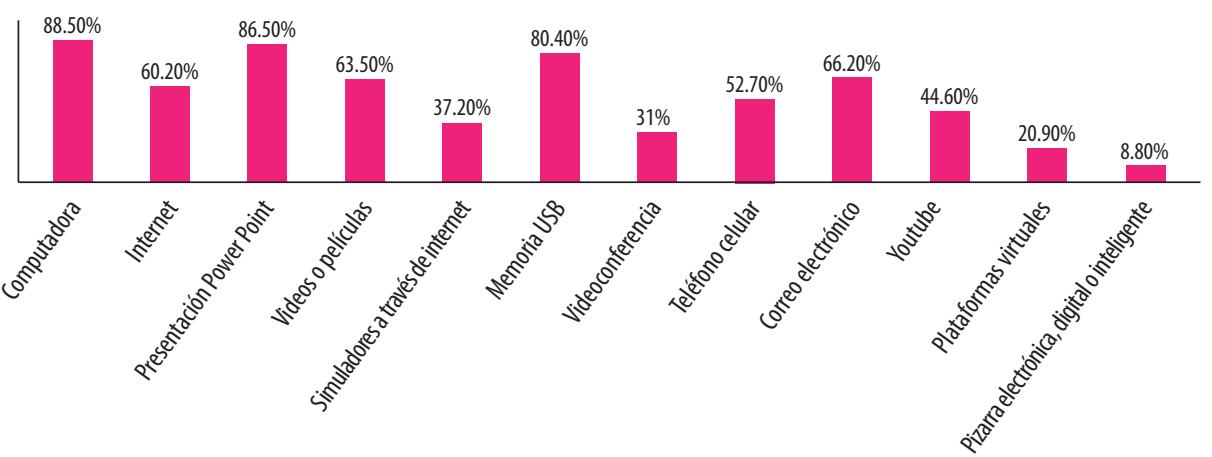

Figura 1. Opinión del estudiante sobre los tipos de recursos tecnológicos que usan los docentes. Fuente: elaboración propia. 
URL: http://www.revistas.una.ac.cr/index.php/dialogo/index

CORREO ELECTRÓNICO: universidadendialogo@una.cr

DOI: https://doi.org/10.15359/udre.10-1.7

Actividades que desarrolla el docente en el aula con ayuda de las TIC

\section{El computador}

Las actividades que realizan los docentes con el computador fueron identificadas por estudiantes y docentes mediante encuesta, quienes coinciden en que se utiliza principalmente para realizar presentaciones en Power Point. Los estudiantes manifiestan que es muy poco usado para la realización de videoconferencias y presentación de videos, lo que fue comprobado durante la observación del aula, donde solo el $14 \%$ de los docentes utilizaron el computador para mostrar videos y realizar videoconferencia.

Así mismo se observó la coincidencia de docentes y estudiantes en el poco uso del computador para aplicar programas interactivos o software educativo. De manera que se está perdiendo una fuente valiosa de interactividad con los estudiantes que les permita la realización de diversas actividades donde se promueva el aprendizaje autónomo e independiente, así como la creación de situaciones de aprendizaje similares a la vida real. Esto coincide con lo expresado por Tejedor y Valcarcel (1996), quienes afirman que la incorporación de las tecnologías de la información y la comunicación (video, informática, telecomunicaciones) es considerada como un medio para superar la barrera entre la teoría y la práctica.

Komoski, citado por Squires y McDougall (1994), dice que el buen uso de los gráficos y el texto en el software pueden aportar a profesores y estudiantes una flexibilidad y control sin precedente y que el mejor software educativo puede extender el alcance, la profundidad y la adecuación de las opciones de enseñanza y aprendizaje más allá de las que tradicionalmente se consiguen en el aula. Sin embargo, hay que considerar una serie de factores por los cuales los docentes de la carrera de Optometría Médica no usan el computador para aplicar software educativo y muy pocos lo utilizan para realizar videoconferencias, esta dificultad puede deberse a la escasez de conocimientos sobre el manejo de este tipo de herramientas dentro del aula, a tener más confianza en los métodos tradicionales de enseñanza, o a la complicación para adaptar los contenidos a las nuevas estrategias tecnológicas.

\section{El Internet}

Los docentes de la carrera de Optometría Médica están utilizando el Internet principalmente como medio de comunicación, trabajo colaborativo, para buscar $\mathrm{y}$ transmitir información, y es poco usado para realizar actividades en la web. 
Revista Universidad en Diálogo • Vol. 10, N. ${ }^{\circ}$ 1, Enero-Junio, 2020 • 109-126

ISSN 2215-2849 • EISSN: 2215-4752

URL: http://www.revistas.una.ac.cr/index.php/dialogo/index Correo eleCtrónico: universidadendialogo@una.cr

DOI: https://doi.org/10.15359/udre.10-1.7

Durante la visita al aula no se observó el uso del Internet en las actividades realizadas, lo cual puede deberse a la infraestructura tecnológica de las aulas, las cuales no tienen acceso a Internet, lo cual impide que los docentes de la carrera de Optometría Médica realicen diversas actividades de enseñanza, como las de la web 2.0; para suplir esta necesidad recurren al Internet que tienen los estudiantes en sus celulares, sin embargo, solo unos pocos cuentan con este servicio en su teléfono, lo que dificulta el trabajo.

\section{Uso del Power Point}

Las presentaciones que realizan los docentes en Power Point tienen un tamaño de letra adecuado, se utiliza buena combinación de colores y contraste, además de tener una secuencia clara, lógica y coherente, lo que fue constatado durante la observación en las aulas de clase donde se evidencia que los docentes están siguiendo las normas establecidas para elaborar las diapositivas, que deben ser simples y sencillas, fáciles de ver y leer, el tamaño de letra recomendado es de treinta y seis para los títulos y de veintiocho a veinticuatro para el contenido de la presentación, se debe utilizar un solo tipo de letra en toda la presentación, debe existir un buen contraste entre el texto y el fondo.

Por otro lado, se observó que en las presentaciones de Power Point que realizan los docentes el recurso multimedia es poco utilizado, lo cual puede generar menos motivación e interés en los estudiantes.

\section{Uso del teléfono celular}

El teléfono celular es utilizado por docentes y estudiantes con mayor frecuencia para la comunicación colectiva, en este sentido, en la entrevista realizada los profesores manifiestan que usan el teléfono celular para acceder a redes sociales como Facebook y WhatsApp, con las cuales se mantienen en comunicación con los estudiantes y les permite compartir información, realizar tareas en clases y bajar aplicaciones de Play Store con las cuales han realizado videos.

Sin embargo, el teléfono celular tiene mayores aplicaciones que benefician el aprendizaje de los estudiantes, los docentes pueden acceder a plataformas donde organicen foros de discusión, así mismo, planificar evaluaciones en clase a través del celular, pero muy pocos docentes lo utilizan para realizar evaluaciones, asignar calificaciones y organizar foros de discusión.

El teléfono celular puede convertirse en una herramienta útil en el proceso de enseñanza. De acuerdo con lo planteado por Galaviz (2014), dentro de las 
URL: http://www.revistas.una.ac.cr/index.php/dialogo/index

CORREO ELECTRÓNICO: universidadendialogo@una.cr

DOI: https://doi.org/10.15359/udre.10-1.7

aplicaciones del celular en un ambiente educativo se encuentran: el correo electrónico o acceso a portales de Internet, descargar material de apoyo para la formación y educación. Existen también una gran variedad de aplicaciones (APPS) que se pueden utilizar en los celulares "inteligentes" y que tienen objetivos educativos o de apoyo a la docencia para diversas plataformas, ya sea IOS, Android, Blackberry o Windows Phone.

\section{El video}

El video es usado por los docentes de la carrera de Optometría Médica principalmente para transmitir información, realizar demostraciones y como apoyo a las explicaciones. Con menor frecuencia, es usado para que los estudiantes expresen sus opiniones, para motivar y para analizar la información presentada.

Así mismo, se encontró que el video no se está utilizando para evaluar ni para realizar tareas individuales o grupales. (Ver tabla 2)

Tabla 2

Actividades encontradas con el uso del video

\begin{tabular}{|l|l|l|}
\hline \multicolumn{1}{|c|}{ Entrevista a docentes } & $\begin{array}{c}\text { Grupo focal con } \\
\text { estudiantes }\end{array}$ & \multicolumn{1}{c|}{ Observación a clase } \\
\hline Apoyo a las explicaciones & Demostraciones & Apoyo a las explicaciones \\
\hline $\begin{array}{l}\text { Realizar tareas con los estudiantes } \\
\text { individual o colectivamente }\end{array}$ & & Hacer demostraciones \\
\hline & & Transmitir información \\
\hline
\end{tabular}

Fuente: elaboración propia

En este sentido, deben buscarse estrategias de utilización del video que proporcionen una acción colectiva y no individual sobre el material; es decir, procurar con su utilización la participación activa del estudiante sobre el documento. Esto concuerda con Cabero et al. (2005), que indican que el video puede ser utilizado en diferentes momentos del proceso de enseñanza. Al principio, para motivar, en medio del acto didáctico o al final del mismo para aclarar conceptos. Facilita la realización de diversas actividades con los estudiantes que van desde las destinadas a complementar la información recibida hasta aquellas que permiten la profundización de la información presentada, de manera que al finalizar el video este se debe relacionar con una actividad inmediata, permitiéndoles a los estudiantes interiorizar y trabajar los contenidos. 


\section{Plataformas virtuales y pizarra digital}

Según la encuesta realizada a docentes y estudiantes, así como la observación realizada en el aula de clase, se comprueba que tanto las plataformas virtuales como la pizarra digital son recursos tecnológicos que no están siendo utilizados por los profesores en las asignaturas que imparten, con lo cual se está perdiendo una gran variedad de recursos que facilitan y mejoran el proceso de aprendizaje.

Valenzuela et al. (2010) afirman que dentro de las principales modalidades de TIC que existen para ser usadas en educación, tanto a distancia como semipresencial, están las plataformas de enseñanza virtuales. Estas han tenido un enorme desarrollo en el último tiempo, ya que permiten crear un entorno virtual de aprendizaje con mucha facilidad, sin necesidad de ser expertos en programación.

\section{Beneficios que promueven las tecnologías de la información y la comunicación en el aprendizaje de los estudiantes}

En la encuesta realizada a estudiantes, estos opinaron que las principales habilidades que promueven las TIC para mejorar el proceso de enseñanza son: favorece la comprensión del tema $85 \%$, mejora la asimilación de contenidos de aprendizaje $80 \%$, favorece la realimentación del tema $77 \%$, facilita la comunicación entre profesores y estudiantes $74 \%$ y en menor porcentaje la reflexión, la atención individualizada y el trabajo colaborativo.

Los estudiantes por su parte tienen una percepción positiva respecto a las TIC, el $70 \%$ afirma que las TIC favorecen positivamente su aprendizaje con la utilización del data show, el cual les permite ver de manera más resumida la clase y comprender mejor, de igual forma la utilización del video les ayuda a asimilar el contenido. (Ver figura 2)

¿Considera que el uso que les da el docente a las TIC favorece su aprendizaje?

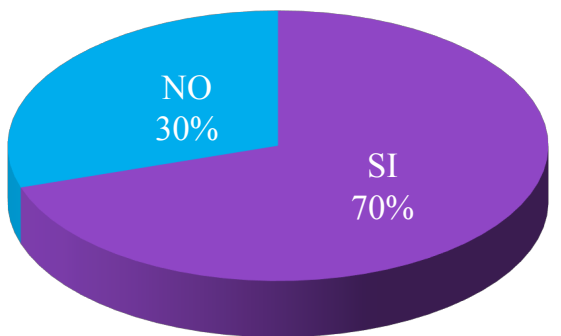

Figura 2. ¿Considera que el uso que les da el docente a las TIC favorece su aprendizaje? 
URL: http://www.revistas.una.ac.cr/index.php/dialogo/index

CORREO ELECTRÓNICO: universidadendialogo@una.cr

DOI: https://doi.org/10.15359/udre.10-1.7

\section{Condiciones que favorecen el uso de las tecnologías de la información y la comunicación en los docentes de la carrera de Optometría Médica}

\section{Infraestructura tecnológica con que cuenta la carrera de Optometría Médica}

Existen diversos factores que influyen en la utilización de las TIC por parte de los profesores, un aspecto de gran importancia lo constituye la infraestructura tecnológica y el acceso que tengan los docentes a esta.

En este sentido, la Facultad de Ciencias Médicas cuenta con un laboratorio de informática para atender a los estudiantes y docentes de la carrera de Optometría Médica, este laboratorio consta de dos salas, en la primera están conectados quince ordenadores y en la segunda veinte, ambas salas tienen conectividad a Internet; sin embargo, para hacer uso de la sala hay que solicitarla con previa anticipación al ingeniero encargado del laboratorio, ya que es solicitado por muchos docentes de otras carreras.

Por su parte, la carrera de Optometría Medica también cuenta con sus propios recursos tecnológicos, dentro de los que figuran: computadoras, data show, parlantes e Internet para los docentes; no obstante, tanto el coordinador de la carrera como los profesores manifestaron que los recursos tecnológicos con que cuenta la carrera son insuficientes para poder aplicar las TIC en el aula.

Uno de los profesores expresó: "Hacen falta herramientas para el desarrollo adecuado de las asignaturas, que se verían optimizadas con el uso de las TIC". Por su lado los estudiantes manifestaron en la entrevista realizada al grupo focal que es importante la inversión que se haga en TIC, porque en las aulas no hay acceso a Internet, lo cual es fundamental para que los profesores implementen la tecnología.

En la observación realizada se constató que las aulas de la carrera de Optometría Médica no cuentan con recursos tecnológicos instalados, como data show, televisores, pizarras digitales o interactivas, así mismo la conexión a Internet inalámbrico es inexistente. Esto coincide con Rhonda y Bates (2004), quienes afirman que se debe contar con una infraestructura tecnológica que apoye la implementación de las TIC por parte de los docentes, como son el acceso a la red de todo el personal docente, instalaciones eléctricas, fibra óptica, ordenadores para cada docente instalados en red, de manera que se establezca una conexión universal en red de la cual se derivan un conjunto de servicios vitales que el docente puede utilizar como medio de enseñanza. 
Revista Universidad en Diálogo • Vol. 10, N. ${ }^{\circ}$ 1, Enero-Junio, 2020 • 109-126

ISSN 2215-2849 • EISSN: 2215-4752

URL: http://www.revistas.una.ac.cr/index.php/dialogo/index CORREO ELECTRÓNICO: universidadendialogo@una.cr DOI: https://doi.org/10.15359/udre.10-1.7

Conocimiento sobre programas, aplicaciones y herramientas que tienen los docentes de las TIC

En las entrevistas realizadas los docentes de la carrera de Optometría Medica manifiestan que tienen mucho conocimiento en el sistema operativo Windows y hojas de cálculo Excel, sin embargo, hay poco conocimiento en editor de audio o video, Publisher y diseño gráfico.

En relación con las aplicaciones TIC, estos refieren tener mucho o suficiente conocimiento en Power Point, YouTube, buscadores de Internet, correo electrónico, mensajería instantánea, redes sociales y Google Drive; sin embargo, se evidencia poco o ningún conocimiento en las plataformas virtuales y herramientas web 2.0. Las herramientas tecnológicas en las que mayor dominio y conocimiento tienen los docentes son: Internet, memoria USB, teléfono celular, computadora, videoconferencia, video educativo, televisor y data show. Refieren tener poco conocimiento de la pizarra digital y los simuladores.

\section{Capacitaciones recibidas por los docentes sobre TIC}

En la entrevista realizada a los docentes, coinciden en que es fundamental la capacitación sobre las TIC, la mayoría manifiestan no haber recibido capacitaciones.

A pesar de la poca o ninguna capacitación que han tenido los docentes de la carrera de Optometría Médica, estos tienen una actitud positiva ante las TIC y aceptan su uso en la práctica pedagógica.

\section{Propuesta de software educativo para la carrera de Optometría Médica}

Con base en los resultados obtenidos de esta investigación, se considera necesario que los docentes hagan uso de plataformas virtuales y simuladores que puedan facilitar a los estudiantes la asimilación de contenidos. De manera que se propone la realización de un software educativo, con el cual los docentes puedan acceder a diferentes recursos tecnológicos; en este se incorporan las asignaturas que imparten los docentes de la carrera de Optometría Médica, con el fin de compartir e interactuar con los estudiantes.

El Software Educativo de Optometría Médica (SE-OM) consta de diversos recursos como videos, libros, artículos científicos, tareas y simuladores. De manera que el docente pueda poner a disposición del estudiante todos los recursos que necesita en las distintas materias de la carrera de Optometría Médica en un solo lugar. La característica de esta plataforma es que es de fácil manejo y puede ser utilizada desde los teléfonos celulares, permitiendo a docentes y estudiantes que realicen las clases de manera más dinámica. 
URL: http://www.revistas.una.ac.cr/index.php/dialogo/index

CORREO ELECTRÓNICO: universidadendialogo@una.cr

DOI: https://doi.org/10.15359/udre.10-1.7

Para la implementación del software es necesario un administrador, el cual regula el acceso a los recursos del sistema mediante la asignación de roles, con los atributos correspondientes. El administrador tiene todos los atributos o derechos dentro del sistema.

\section{División de los roles}

Profesor de planta. Tiene asignados derechos como: subir, borrar y modificar información, formar grupos, agregar participantes a los grupos organizados.

Profesor horario. Tiene casi los mismos derechos que tienen los profesores de planta, salvo en las cantidades de información y algunos datos que no son accesibles a ellos.

Estudiantes. Tienen menos derechos dentro del uso del sistema. Pero pueden tener acceso a la información sin modificarla. Subir archivos bajo la supervisión de un docente. Participar en trabajos colaborativos.

Otra de las funciones del administrador es el manejo de la base de datos de los nombres de usuarios y las contraseñas, la actualización de las mismas, el envío a los correos de los participantes del sistema, el manejo de las bases de datos de calificaciones, el manejo de las bases de datos de los datos personales de los participantes, el ingreso de la información para la actualización del sistema con la colaboración de los maestros. Para ello debe manejar los ambientes de trabajo: Macromedia Dreamweaver, Macromedia Flash, el sistema de gestión de bases de datos SQL y el lenguaje HTML.

El administrador entra al sistema y cambia la programación de las páginas web, utilizando lenguajes de cara al servidor o de cara al cliente. Además, páginas de estilo en cascada, las cuales permiten definir el estilo de los sitios en los encabezados de las páginas, antes del body.

El administrador entra al mismo con el editor de sitios web, o con el ambiente de programación de páginas web, luego pasa de la presentación de diseño a la presentación de los códigos, combina estos para actualizar los contenidos. La mayoría de las instrucciones son a base de etiquetas o label, también se utilizan scripts o guiones para determinar las instrucciones, por ello debe conocer todos los formatos de los comandos de la programación HTML, pero, además, debe conocer los comandos de los lenguajes de apoyo o complementos a las páginas web dinámicas, tales como ASP o PHP. También JavaScript y Visual Basic Script.

Se sugiere dar un pequeño entrenamiento al administrador del Software Educativo de Optometría Médica (SEOM) para que pueda cumplir con la organi- 
Revista Universidad en Diálogo • Vol. 10, N. ${ }^{\circ}$ 1, Enero-Junio, 2020 • 109-126

ISSN 2215-2849 • EISSN: 2215-4752

URL: http://www.revistas.una.ac.cr/index.php/dialogo/index CoRREO ELECTRÓNICO: universidadendialogo@una.cr DOI: https://doi.org/10.15359/udre.10-1.7

zación, el funcionamiento y la seguridad necesaria para el buen funcionamiento y escalabilidad del sistema web.

\section{Conclusiones}

Los docentes de la carrera de Optometría Médica están incorporando las TIC en el proceso de enseñanza que realizan con los estudiantes, los recursos más utilizados son: computadora, Power Point, data show, memoria USB, videos de YouTube y redes sociales; recursos de más fácil manejo y a los cuales tienen mayor acceso. Siendo menos usados los simuladores, las pizarras digitales, las plataformas virtuales y las videoconferencias, recursos que requieren una mayor inversión, conocimiento y manejo por parte de los docentes.

La infraestructura tecnológica con que cuenta la carrera de Optometría Médica está constituida por equipos básicos como data show, parlantes, extensiones, computadoras e Internet para los docentes. Sin embargo, estos recursos resultan insuficientes para poder aplicar las TIC en el aula. Para poder aplicar las TIC con todos sus componentes se hace necesario adquirir pizarras inteligentes o digitales, más computadoras, más parlantes, así como el Internet en las aulas.

Los docentes de la carrera de Optometría Médica no han recibido capacitación en el uso de las TIC como herramienta para favorecer el aprendizaje de los estudiantes. La mayoría tiene conocimientos básicos que los ha aprendido empíricamente. No existe un plan diseñado para la capacitación y la educación continua del profesorado.

Los estudiantes manifiestan que el uso de las TIC favorece el aprendizaje en cuanto a la comprensión del tema, mejora la asimilación de contenidos de aprendizaje, favorece la retroalimentación y facilita la comunicación entre profesores y estudiantes, la reflexión y el debate por el estudiante, atención individualizada y trabajo colaborativo, sin embargo, estas no están siendo desarrolladas por los docentes por la falta de actividades o estrategias metodológicas implementadas.

De manera que podemos concluir que para aprovechar los innumerables beneficios que ofrecen las TIC en el proceso educativo existen varios factores que se deben superar si queremos lograr su adecuada implementación; quizás uno de los más importantes es la disposición y conocimientos que tengan los docentes en el uso de las TIC, así como la infraestructura tecnológica con que se cuente y los planes de formación continua que se planifiquen en estrategias metodológicas usando las TIC. 


\section{Referencias bibliográficas}

Cabero, J. (1998). Impacto de las nuevas tecnologías de la información y la comunicación en las organizaciones educativas. En M. Lorenzo y otros (Coords.), Enfoques en la organización y dirección de instituciones educativas formales y no formales (pp. 197-206). Granada: Grupo Editorial Universitario. http://tecnologiaedu. .us.es/bibliovir/pdf/75.pdf.

Cabero, J., Llorente, M., Román, P. (2005). Las posibilidades del vídeo digital para la formación. Universidad de Sevilla, España-UE.

Coll, C. (2011). Aprender y enseñar con las TIC: expectativas, realidad y potencialidades. R. Carneiro, J. Toscano y Díaz (Eds.), Los desafíos de las TIC para el cambio educativo (pp. 112-125). Madrid, España: Fundación Santillana.

Fernández, I. (2010). Las TIC en el ámbito educativo. Revista Electrónica EDUCREA. https://educrea.cl/las-tics-en-el-ambito-educativo/

Ferreiro, R. (2010). El ABC del aprendizaje cooperativo: trabajo en equipo para aprender y enseñar. México: Editorial Trillas.

Tejedor, F. G. y Valcarcel, A. G. (1996). Perspectivas de las nuevas tecnologías en la educación. Madrid, España: Edición Narcea.

García, K. S. (2012). Rol del docente y del estudiante universitario en el uso de las tecnologías de información como recursos didácticos en el proceso enseñanza-aprendizaje del tercer año de la carrera de Pedagogía con Mención en Educación (tesis de Máster en Pedagogía, Universidad Nacional Autónoma de Nicaragua, UNAN Managua). http://repositorio. unan.edu.ni/1191/

Galaviz, J. M. (2014). Análisis comparativo en el uso de las TIC para aplicaciones educativas de la competencia tecnológica Tlatemoani. Revista Grupo Eumed.net (Universidad de Málaga), (15). Recuperado de http://www.eumed.net/rev/tlatemoani/index.htm

Hernández, L., Acevedo, J. A. S., Martínez, C. y Cruz, B. C. (2014). El uso de las TIC en el aula: un análisis en términos de efectividad y educación. Congreso Iberoamericano de Ciencia, Tecnología, Innovación y Educación. Buenos Aires, Argentina. https://es.scribd.com/document/351901034/tic-pdf

Hernández, R., Fernández, C. y Baptista, P. (2006). Metodología de la investigación (cuarta edición). México: McGraw-Hill Interamericana. 
Marqués, P. (2008). La pizarra digital. Departamento de Pedagogía Aplicada, Facultad de Educación, UAB. http://peremarques.pangea.org/pdigital/ es/pizinteractiva.htm\#ventaja

Pulido, W. y Zambrano, J. (2010). Capítulo 9: Uso de recursos educativos abiertos para comprender las características de las gráficas de funciones de dos variables. En M. S. Ramírez Montoya y J. V. Burgos Aguilar (Coords.), Recursos educativos abiertos enriquecidos con tecnología. Innovación en la práctica educativa (pp. 164-182). México: Tecnológico de Monterrey (ITESM). https://coleccion.siaeducacion.org/node/1605

Ruiz, E. A. (2012). Análisis de la incidencia de las tecnologías informáticas de la computación, en el proceso de enseñanza-aprendizaje que realiza el docente de Ciencias Sociales, con los alumnos de segundo año de magisterio de la Escuela Normal "Mirna Mairena Guadamuz" del municipio de Esteli (tesis de Maestría). Universidad Nacional Autónoma de Nicaragua, Managua. http://repositorio.unan.edu.ni/1191/

Rhonda, M. y Bates, A. W. (2004). Enseñar al profesorado cómo utilizar la tecnología. Barcelona, España: Editorial UOC.

Squires, D. y McDougall, A. (1994). Cómo elegir y utilizar software educativo. Guia para el profesorado. Madrid, España: Ediciones Morata.

UNAN. (2011). Modelo Educativo Normativo y Metodología para la Planificación Curricular. Universidad Nacional Autónoma de Nicaragua, Managua, Nicaragua. http://pagines.uab.cat/unan_uab_innovadocencia/ sites/pagines.uab.cat.unan_uab_innovadocencia/files/Modelo_ Educativo19_de_septiembre.p̄ $\overline{\mathrm{d} f}$

Unesco (2013). Enfoques estratégicos sobre las TIC en educación en América latina yelCaribe. http://www.unesco.org/new/fileadmin/MULTIMEDIA/ FIELD/Santiago/images/ticsesp.pdf

Valenzuela, J., Bellei, C. y De los Ríos, D. (2010). Segregación escolar en Chile. Chile: Unesco y Pontificia Universidad de Chile.

Vélez, C. I. (2012). Estrategias de enseñanza implementadas por los docentes de Básica Secundaria y Media Técnica de la Institución Educativa Técnico Industrial Pedro Castro Monsalvo, 8- Valledupar, al utilizar las TIC en su práctica pedagógica para favorecer el aprendizaje significativo de los estudiantes (tesis de Maestría). Universidad Virtual Escuela de Graduados en Educación, Tecnológico de Monterrey, México. 